\title{
Relación entre la comprensión lectora y las estrategias de aprendizaje en estudiantes de secundaria en un distrito de Lima
}

\author{
Alberto Alegre Bravo \\ Universidad de Lima (Perú)
}

Recibido: 2 de julio del 2009 / Aprobado: 30 de julio del 2009

Se estudió la relación entre la comprensión lectora y las estrategias de aprendizaje en alumnos de quinto grado de secundaria de colegios estatales del distrito de Independencia, en Lima. Se utilizó la Prueba Cloze de Lectura (González \& Quesada, 1997) y la Escala de Estrategias de Aprendizaje - ACRA (Román \& Gallego, 1994). En el análisis de la contrastación de hipótesis se encontró que no existe relación entre la comprensión lectora y las estrategias de aprendizaje. Asimismo, estos estudiantes se ubican mayoritariamente en el nivel de comprensión lectora Deficitario e Intermedio en cada una de las estrategias de aprendizaje.

lectura / comprensión lectora / estrategias de aprendizaje

\section{Relationship between reading comprehension and learning strategies on secondary school students in a district of Lima}

Relationship between reading comprehension and learning strategies was investigated in fifth grade secondary school students of public schools of Independencia District, Lima. We used the Cloze Reading Test (González \& Quesada, 1997) and the Learning Strategies Scale - ACRA (Román \& Gallego, 1994). After the hypothesis constrasting analysis it was found no correlation between reading comprehension and learning strategies. Likewise, these students are in the Deficient level in Reading Comprehension and Intermediate level on each of learning strategies.

reading / reading comprehension / learning strategies

Correo electrónico: aalegre@ulima.edu.pe 


\section{INTRODUCCIÓN}

La lectura es considerada una competencia fundamental que adquieren y desarrollan los niños y los adolescentes durante el proceso de enseñanza-aprendizaje en la educación básica, ya que es un medio imprescindible para acceder a la sociedad del conocimiento.

En el sistema educativo de nuestro país, el interés y la preocupación por la lectura han estado centrados principalmente en su función como medio en el proceso de aprendizaje, propiciando que su enseñanza haya incidido especialmente en la decodificación, así como en la fluidez y la velocidad de lectura, descuidando aspectos de comprensión. A pesar de los diversos esfuerzos desplegados en los últimos años en mejorar la comprensión de lectura en el estudiante peruano de educación secundaria, esta aún se mantiene significativamente por debajo del nivel esperado. Esta pobreza ha sido puesta en evidencia por los resultados de las evaluaciones nacionales de rendimiento escolar de los años 1996, 1998, 2001 y 2004 (Cueto, 2007), así como en las evaluaciones internacionales de Llece (Llece, 2001) y Pisa (OCD-Ince, 2001). En esta última, comparados con estudiantes de más de cuarenta países, los peruanos obtuvieron el último lugar en Alfabetización Lectora.

Ante esta problemática, la Dirección Nacional de Educación Superior y Tecnológica (2006) en la nueva propuesta curricular plantea que en primaria se enseñen estrategias de comprensión de lectura que ayuden a interpretar el texto; la meta es el aprendizaje de la comprensión lectora. En secundaria el fin es distinto: enseñar estrategias que permitan que el estudiante lea para aprender. En este nivel la meta es aprender contenidos y la comprensión de lectura cumple la función de instrumento para llegar a ese fin.

Las estrategias de aprendizaje, por otro lado, son muy importantes en la medida en que incluyen recursos cognitivos, así como elementos directamente vinculados con la disposición y la motivación del estudiante para las actividades de planificación, dirección y control, puestas en marcha por el sujeto para hacer frente al aprendizaje. La adquisición de estrategias de aprendizaje, de acuerdo con las investigaciones, se ha enmarcado en un "período crítico" que podría ubicarse entre los 11 y los 14 años (Pramling, 1990), siendo fundamental su enseñanza a los alumnos en primaria y secundaria, aunque no está restringida a estos niveles, pues también se puede enseñar en la universidad y hasta en educación infantil, con el objetivo de que cada sujeto se convierta en un estudiante independiente.

Para que los alumnos puedan desarrollar la comprensión de lectura se requieren intervenciones en el ámbito educativo que les proporcione estrate- 
gias en cada uno de los procesos cognitivos (adquisición, codificación y recuperación) implicados en el tratamiento y elaboración de la información brindada por el texto, que permitan al lector construir significados, así como desarrollar los elementos metacognitivos y socioafectivos que sirvan de apoyo a dicho procesamiento, y que son necesarios en el proceso de enseñanzaaprendizaje.

\section{La lectura}

La lectura es una actividad a través de la cual los lectores construyen significados (comprensión); en ella se combinan los conocimientos y las experiencias previas, la competencia lingüística (fonológica, léxica, información sintáctica, semántica), la información aportada por el texto y el contexto, y la forma como se relacionan con el texto (de acuerdo a la gramática textual). Es, por lo tanto, un proceso cognitivo, psicolingüístico y sociocultural (Tapia, 2008). Esta actividad comprende una serie de procesos, los cuales se desarrollan a continuación:

- Procesos perceptivos: Este proceso se encarga de transformar la información impresa en algún tipo de código viso-espacial, almacenando estos inputs transformados brevemente (solo unos milisegundos) en la memoria sensorial de tipo icónica, permitiendo así que la memoria operativa seleccione y trate los ras- gos más relevantes para reconocerlos como unidades lingüísticas, como representaciones ortográficas. $\mathrm{Al}$ igual que con la percepción del habla, la percepción del lenguaje escrito comprende varios niveles de procesamiento: rasgos, letras y palabras (Carroll, 2006).

- Procesamiento léxico: Una vez identificadas las unidades lingüísticas, el siguiente proceso es la transformación de esas unidades ortográficas en conceptos, al procesarlas desde el conocimiento previo almacenado en el léxico interno o "lexicón" del lector. Para llegar hasta el significado almacenado en el sistema semántico a partir de la palabra escrita, existirían dos caminos o rutas alternativas: La ruta léxica o visual y la ruta fonológica (Cuetos, 1991).

- Procesamiento sintáctico: Se encarga del procesamiento de las relaciones (sintácticas y gramaticales) entre las palabras, constituyéndose en un componente importante de la lectura, que básicamente comprende la asignación de las etiquetas correspondientes a las distintas áreas de las palabras que componen la oración, la especificación de las relaciones existentes entre estos componentes y la construcción de la lectura correspondiente, mediante el ordenamiento jerárquico de los componentes. 
- Procesamiento semántico: Este es el último de los procesos y consiste en extraer el significado de la oración o texto y de integrarlo con los conocimientos previos que posee el lector, como por ejemplo los esquemas, que vienen a ser modelos internos de las diferentes situaciones con las que se encuentra el lector, y que además posibilitan la elaboración de las inferencias necesarias para la comprensión.

\section{La comprensión lectora}

La comprensión de lectura de un texto es la reconstrucción de su significado a partir de la consideración de múltiples pistas contenidas en ese texto, dadas por el autor y mediante la interacción con los conocimientos previos del lector (Morles, 1999).

En este proceso de construcción del significado se requiere que el lector sea capaz de procesar las palabras y las oraciones que le propone el texto y de involucrar sus conocimientos del mundo, esquemas y propósitos, así como la forma en que el autor ha organizado sus ideas, que es lo que constituye la estructura del texto (González, 1996).

Para Perfetti (Escurra, 2002) la comprensión de lectura presenta cuatro niveles:

- Nivel superficial: Supone que el lector es capaz de determinar las propiedades fonológicas y acústicas más sobresalientes del texto.
- Nivel de comprensión básico: Este nivel requiere que exista una competencia lingüística para entender la oración que se lee y lograr con eso una cierta descripción.

- Nivel interpretativo: Permite al lector establecer relaciones significativas que van más allá del texto escrito. Supone dominar con mayor amplitud el contexto lingüístico para establecer las inferencias personales. Para que el lector pueda interpretar un texto se requiere analizar el significado de los conceptos generales.

- Nivel de comprensión de lectura crítica o valorativa: Se logra cuando ya se han realizado los niveles anteriores. En este nivel, el sujeto debe ser capaz de ordenar y reordenar los conceptos dentro del texto (tareas realizadas en niveles anteriores) para captar el mensaje del autor de acuerdo con la realidad que se está analizando. Al realizar la lectura, el lector va enriqueciendo y transformando el texto con su propio pensamiento. La lectura crítica implica una participación activa por parte del lector en lo que lee a través de la búsqueda de información que le permita realizar un análisis, ya que para interpretar y valorar se requiere contar con puntos de vista para efectuar comparaciones. Estos puntos de vista pueden ser las experiencias y vivencias del propio lector (Pinzás, 2003). 


\section{Las estrategias de aprendizaje}

Según Monereo (2000), las estrategias de aprendizaje son procesos de toma de decisiones en los cuales el estudiante elige y recupera, de manera coordina$\mathrm{da}$, los conocimientos que necesita para complementar una determinada demanda u objetivo, dependiendo de las características de la situación educativa en que se produce la acción.

Para Genovard y Gotzens (1996) las estrategias de aprendizaje pueden definirse como el conjunto de comportamientos que el alumno despliega durante su proceso de aprendizaje y que, supuestamente, influyen en el proceso de codificación de la información que debe aprender.

Existen coincidencias entre los autores más representativos en este campo en resaltar como elementos importantes del concepto de estrategias de aprendizaje los siguientes: por un lado, las estrategias implican una secuencia de actividades, operaciones o planes dirigidos a la consecución de metas de aprendizaje; y por otro, tienen un carácter consciente e intencional en el que están implicados procesos de toma de decisiones por parte del alumno, ajustados al objetivo o meta que pretende conseguir.

Las estrategias de aprendizaje son procedimientos que se aplican de un modo intencional y deliberado a una tarea y que no pueden reducirse a rutinas automatizadas, es decir, son más que simples secuencias o aglomeraciones de habilidades. Como afirma Beltrán (1996), las estrategias tienen un carácter intencional; implican, por tanto, un plan de acción frente a la técnica, que es marcadamente mecánica y rutinaria.

Si se admite la hipótesis de que los principales aspectos cognitivos del procesamiento de la información son los de adquisición, codificación o almacenamiento, y recuperación, las estrategias cognitivas de aprendizaje o estrategias de procesamiento pueden ser definidas como secuencias integradas de procedimientos o actividades mentales que se activan con el propósito de facilitar la adquisición, almacenamiento y/o utilización de la información (Nisbel \& Shucksmith, 1987).

Román y Gallego (1994), sobre la base de la actual hipótesis admitida de los principales procesos cognitivos de procesamiento de información, plantean que las estrategias cognitivas de aprendizaje o estrategias de procesamiento pueden ser definidas como secuencias integradas de procedimientos o actividades mentales que se activan con el propósito de facilitar la adquisición, almacenamiento y utilización de la información.

Estos autores deducen cuatro tipos de estrategias de procesamiento o estrategias cognitivas para su manipulación (control y dirección). Es decir, procedimientos que permiten optimizar, enseñar, prevenir y corregir su adecuado funcionamiento. Estos procedi- 
mientos mentales o estrategias de manejo, a las que suele denominarse microestrategias, tácticas de aprendizaje o estrategias de estudio, suelen ser públicas privadamente observables por contraposición, a los procesos que son constructos inferidos. Los tipos de estrategias de aprendizaje propuestos por Román y Gallego (1994) son:

- Estrategias de adquisición de información: De acuerdo al modelo de Atkinson y Shiffrin, para adquirir información son necesarios los procesos atencionales, que son los encargados de seleccionar, transformar y transportar la información desde el medio ambiente hasta el registro sensorial. Luego se ponen en marcha los procesos de repetición, encargados de llevar la información (transformarla y transportarla), junto con los atencionales y en interacción con ellos desde el registro sensorial a la memoria a corto plazo. En el ámbito de la adquisición de la información se han constatado dos tipos de estrategias de procesamiento.

- Estrategias de codificación de información: Se refiere al proceso cognitivo encargado de la elaboración y organización de la información, conectándola con conocimientos previos e integrándola en estructuras de significado más amplias, que forman la base del conocimiento, es decir del paso de la memoria de corto plazo a la memoria de largo plazo (MLP).
- Estrategias de recuperación de información: Se refiere a las estrategias que favorecen la búsqueda de información en la memoria y la generación de respuesta.

- Estrategias de apoyo al procesamiento: Durante el tiempo que dura el procesamiento de información, otros procesos de naturaleza metacognitiva y no cognitiva, los de apoyo optimizan, son neutrales o entorpecen el funcionamiento de las estrategias cognitivas de aprendizaje. Por eso los alumnos también necesitan estrategias y tácticas que les ayuden a "manejar" sus estrategias de apoyo. Estas estrategias ayudan y potencian el rendimiento de las estrategias de adquisición, las de codificación y las de recuperación, incrementando la motivación, la autoestima y la atención. Garantizan el clima adecuado para un buen funcionamiento de todo el sistema cognitivo. De ahí que para llevar a cabo el procesamiento y la recuperación de información sea imprescindible su identificación y correcto manejo.

\section{HIPÓTESIS}

Para el desarrollo del estudio se plantearon las siguientes hipótesis:

1. Existe relación entre la comprensión de lectura y el uso de las estrategias de adquisición de información en estudiantes de quinto grado de educación secundaria de cole- 
gios estatales del distrito de Independencia.

2. Existe relación entre la comprensión de lectura y el uso de las estrategias de codificación de información en estudiantes de quinto grado de educación secundaria de colegios estatales del distrito de Independencia.

3. Existe relación entre la comprensión de lectura y el uso de las estrategias de recuperación de información en estudiantes de quinto grado de educación secundaria de colegios estatales del distrito de Independencia.

4. Existe relación entre la comprensión de lectura y el uso de las estrategias de apoyo al procesamiento en estudiantes de quinto grado de educación secundaria de colegios estatales del distrito de Independencia.

\section{MÉTOdo}

El método utilizado fue el descriptivocorrelacional (Hernández, FernándezCollado \& Baptista, 2003), con el propósito de evidenciar y describir las relaciones entre los puntajes de las variables (comprensión de lectura y estrategias de aprendizaje), en los alumnos del quinto grado de secundaria pertenecientes al distrito de Independencia. El diseño fue de tipo descriptivo-correlacional bivariado, buscando determinar la relación entre las variables estudiadas (Sánchez \& Reyes, 2002).

\section{PARTICIPANTES}

La población objetivo estuvo constituida por todos los alumnos de quinto grado de secundaria pertenecientes a los colegios estatales del distrito de Independencia.

La muestra del estudio se obtuvo por un procedimiento intencional no probabilístico, eligiéndose para trabajar alumnos de cinco colegios estatales mixtos con nivel secundaria, que poseían turnos de mañana y tarde.

La muestra total estuvo conformada por 455 alumnos del quinto grado de nivel secundaria; $215(47,3 \%)$ estudiantes pertenecían al sexo masculino y $240(52,7 \%)$ al sexo femenino (véase la tabla 1).

Tabla 1

Composición de la muestra

\begin{tabular}{lrc}
\hline & Frecuencia & Porcentaje \\
\hline Sexo & & \\
Masculino & 215 & 47,3 \\
Femenino & 240 & 52,7 \\
Edad & & \\
15 & 43 & 9,5 \\
16 & 272 & 59,8 \\
17 & 96 & 21,1 \\
18 & 39 & 8,6 \\
19 & 4 & 0,9 \\
20 & 1 & 0,2 \\
Colegio & & \\
1 & 67 & 14,7 \\
2 & 169 & 37,1 \\
3 & 83 & 18,2 \\
4 & 98 & 21,5 \\
5 & 38 & 8,4 \\
\hline
\end{tabular}

$\mathrm{N}=455$ 


\section{Variables de estudio}

- Comprensión lectora: Cuantificada a través de la Prueba Cloze de Lectura que evalúa la comprensión lectora inferencial (González \& Quesada, 1997).

- Estrategias de aprendizaje: Evaluada a través de la Escala de Estrategias de Aprendizaje-ACRA (Román \& Gallego, 1994).

\section{Instrumentos}

\section{Prueba Cloze de Lectura}

Para el desarrollo de la presente investigación se empleó la Prueba Cloze de Lectura, elaborada por González y Quesada (1997), que originalmente estuvo conformada por seis textos y que utiliza el procedimiento "Cloze". Sin embargo, para la presente investigación solo se utilizaron los textos básicos relacionados con los rubros examinados por Barton y Kirsh (1990), alfabetización en prosa, a la que González rotula como texto informativo: "El niño y la familia" (texto 1); alfabetización documental, a la que este denomina texto documentario: "Tipos de hogar en el Perú" (texto 2); y alfabetización numérica, que es el texto numérico (texto 3).

Para calificar la prueba se otorga un punto para cada espacio respondido con una palabra correctamente identificada por texto. El número de respues- tas correctas se divide entre el total de respuestas posibles y el resultado se multiplica por cien, con lo que se obtiene el porcentaje de respuestas correctas (Condemarín \& Milicic, 1998). La evaluación cualitativa permite determinar los niveles de comprensión en cada texto: por encima del $75 \%$ le corresponde un nivel Independiente, de 44$74 \%$ un nivel Dependiente y por debajo del $43 \%$ un nivel Deficitario.

Escala de Estrategias de Aprendizaje ACRA

El uso de las estrategias de aprendizaje se evaluó a través del ACRA Escalas de Estrategias de Estrategias de Aprendizaje (Román \& Gallego, 1994). Se trata de cuatro escalas independientes que evalúan el uso que habitualmente hacen los estudiantes: Escala I, mide las estrategias de adquisición de información: atencionales y de repetición; Escala II, evalúa las estrategias de codificación o almacenamiento de información: mnemóticas, de elaboración y de organización; Escala III, mide las estrategias de recuperación de información: búsqueda y generación de respuesta; y Escala IV, evalúa las estrategias de apoyo al procesamiento: autoconocimiento, automanejo/planificación y automanejo/regulación/evaluación, autoinstrucciones, autocontrol, contradistractoras, interacciones sociales, motivación intrínseca y extrínseca; y motivación de escape. 
Los enunciados se califican en un sistema tipo Likert, con cinco puntos de calificación, que van desde Nunca (0) hasta Siempre (5). Los puntajes de cada escala se obtienen sumando los ítems respectivos. La escala puede ser aplicada de manera individual o colectiva y está diseñada para ser administrada a grupos de adolescentes y adultos por el tipo de afirmación que contiene. El tiempo de duración de la aplicación del instrumento es aproximadamente de 50 minutos.

\section{RESULTADOS}

\section{Análisis descriptivo}

Los resultados del análisis de la bondad de ajuste a la curva normal, realizado a través de la prueba de KolmogorovSmirnov (véase la tabla 2), indican que los textos de la Prueba de Comprensión
Lectora no tiene una distribución normal, mientras que las cuatro escalas del ACRA sí presentan dicha distribución. Debido a estos resultados, los análisis estadísticos de los datos son del tipo no paramétrico.

\section{Contraste de hipótesis}

Inicialmente se planteó la hipótesis teórica, la cual propuso que existe relación entre la comprensión lectora y las estrategias de aprendizaje en los alumnos de quinto grado de secundaria en colegios estatales del distrito de Independencia. Para contrastarla se efectuó el análisis a través del Coeficiente de Correlación de Spearman (rho).

Al analizar la tabla 3 se puede observar que la comprensión lectora (puntuaciones en los tres textos) en los alumnos, así como las estrategias de adquisición de la información tienen

Tabla 2

Análisis de la bondad de ajuste a la curva normal de las variables estudiadas

\begin{tabular}{llrrc}
\hline Variables & & M & D.E. & $\begin{array}{c}\text { Z de } \\
\text { Kolmogorov- Smirnov }\end{array}$ \\
\hline Comprensión lectora & Texto I & 34.94 & 17.27 & 1.57 \\
& Texto II & 38.75 & 17.99 & 1.39 \\
& Texto III & 29.50 & 15.12 & 1.90 \\
\hline \multirow{3}{*}{ Estrategias de aprendizaje } & & & & \\
& Escala I & 46.90 & 7.68 & 1.23 \\
& Escala II & 110.61 & 18.79 & .84 \\
& Escala III & 48.63 & 8.82 & 1.07 \\
& Escala IV & 95.56 & 16.52 & .68 \\
\hline
\end{tabular}

$\mathrm{N}=455$ 
correlación baja, siendo a su vez una de ellas negativa, como es el caso de la correlación entre el texto documentario y las estrategias de adquisición $(r=-$ 0.039), no siendo estas significativas. Por tanto, se concluye que la primera hipótesis, en la cual se planteó que existe relación entre la comprensión de lectura y el uso de las estrategias de adquisición de la información aprendizaje en los estudiantes de quinto grado de educación secundaria de colegios estatales del distrito de Independencia, no se puede aceptar.

Tabla 3

Correlación de Spearman entre los textos de comprensión lectora y las estrategias de adquisición de la información

\begin{tabular}{lc}
\hline & $\begin{array}{c}\text { Estrategias de } \\
\text { adquisición de la } \\
\text { información } \mathbf{r}\end{array}$ \\
\hline Texto informativo & 0.091 \\
Texto documentario & -0.039 \\
Texto numérico & 0.038 \\
\hline
\end{tabular}

$\mathrm{N}=455$

En los alumnos de quinto grado de secundaria las correlaciones entre las puntuaciones en los textos (comprensión lectora) y las estrategias de codificación de la información son bajas ( $\mathrm{r}=$ $0.074, r=0.050)$, negativas $(-0.003) \mathrm{y}$ no significativas (véase la tabla 4), lo cual indica que la segunda hipótesis específica no se acepta, ya que no existe relación entre las variables estudiadas.
Tabla 4

Correlación de Spearman entre los textos de comprensión lectora y las estrategias de codificación de la información

\begin{tabular}{lc}
\hline & $\begin{array}{c}\text { Estrategias de } \\
\text { codificación de la } \\
\text { información } \mathbf{r}\end{array}$ \\
\hline Texto informativo & 0.074 \\
Texto documentario & -0.003 \\
Texto numérico & 0.059 \\
\hline
\end{tabular}

$\mathrm{N}=455$

Las relaciones entre la comprensión lectora (puntuaciones de los textos) y las estrategias de recuperación de la información en los alumnos (véase la tabla 5), son significativas solo en el caso de dos textos (informativo y numérico) pero es baja; por ello, la tercera hipótesis no es aceptada.

Tabla 5

Correlación de Spearman entre los textos de comprensión lectora y las estrategias de recuperación de la información

\begin{tabular}{lc}
\hline & $\begin{array}{c}\text { Estrategias de } \\
\text { recuperación de la } \\
\text { información } \mathbf{~}\end{array}$ \\
\hline Texto informativo & 0.141 \\
Texto documentario & 0.031 \\
Texto numérico & 0.116 \\
\hline
\end{tabular}

$\mathrm{N}=455$

La cuarta hipótesis, en la que se establece relación entre la comprensión lectora y las estrategias de apoyo al procesamiento de la información, no es aceptada; en la tabla 6 se observa que 
la asociación entre ambas variables es baja y no significativa.

Tabla 6

Correlación de Spearman entre los textos de comprensión lectora y las estrategias de apoyo al procesamiento de la información

\begin{tabular}{lc}
\hline & $\begin{array}{c}\text { Estrategias de apoyo } \\
\text { al procesamiento } \\
\text { de la información r }\end{array}$ \\
\hline Texto informativo & 0.074 \\
Texto documentario & 0.056 \\
Texto numérico & 0.087 \\
\hline
\end{tabular}

$\mathrm{N}=455$

\section{Análisis complementario}

En la tabla 7, que muestra los tipos de lector, se puede apreciar que el mayor porcentaje de alumnos se ubica en el ni- vel lector deficitario en los tres textos (informativo $=67,3 \%$, documentario $=$ $58,0 \%$, numérico $=78,9 \%$ ); el nivel lector dependiente en los mismos textos corresponde al 30,5\% (texto 1), 39,6\% (texto 2) y $20,9 \%$ (texto 3 ). Solo han alcanzado el nivel lector independiente el 2,2\% en el primer texto, el 2,4\% en el segundo y el $0,2 \%$ en el tercero.

En la tabla 8 se presenta la distribución de frecuencias y los porcentajes correspondientes a los niveles alcanzados por los alumnos de quinto grado de secundaria en las estrategias de aprendizaje, observándose que el mayor porcentaje se ubica en el nivel intermedio en adquisición, codificación, recuperación y apoyo al procesamiento de la información, es decir, en el 53,8\%, $49,9 \%, 50,3 \%$ y $49,0 \%$, respectiva-

Tabla 7

Niveles de comprensión de lectura

\begin{tabular}{llcr}
\hline Comprensión lectora & \multicolumn{1}{c}{ Niveles } & f & \% \\
\hline Texto & Deficitario & 306 & 67.3 \\
informativo & Dependiente & 139 & 30.5 \\
& Independiente & 10 & 2.2 \\
Texto & Deficitario & 264 & 58.0 \\
documentario & Dependiente & 180 & 39.6 \\
& Independiente & 11 & 2.4 \\
Texto & & 359 & 78.9 \\
numérico & Deficitario & 95 & 20.9 \\
& Dependiente & 1 & 0.2 \\
\hline & Independiente & 455 & 100 \\
\hline
\end{tabular}

$\mathrm{N}=455$. 
Tabla 8

Niveles de estrategias de aprendizaje

\begin{tabular}{llcc}
\hline Estrategias de aprendizaje & Niveles & $\mathbf{f}$ & $\%$ \\
\hline Adquisición de la información & Inferior & 121 & 26.6 \\
& Intermedio & 245 & 53.8 \\
& Superior & 89 & 19.6 \\
Codificación de la información & & & \\
& Inferior & 119 & 26.2 \\
& Intermedio & 227 & 49.9 \\
& Superior & 109 & 24.0 \\
Recuperación de la información & Inferior & 114 & 25.1 \\
& Intermedio & 229 & 50.3 \\
Apoyo al procesamiento de la información & Superior & 112 & 24.6 \\
& & & \\
& Inferior & 117 & 25.7 \\
& Intermedio & 223 & 49.0 \\
& Superior & 115 & 25.3 \\
\hline
\end{tabular}

$N=455$

mente. En el nivel inferior se ubican el $26,6 \%$ en adquisición, el $26,2 \%$ en codificación, el $25,1 \%$ en recuperación y el $25,7 \%$ en apoyo al procesamiento. Solo alcanzaron puntuaciones correspondientes al nivel superior el 19,6\% en el primer tipo de estrategias, el $24,0 \%$ en el segundo, el $24,6 \%$ en el tercero y el $25,3 \%$ en el cuarto.

\section{DISCUSIÓN}

El análisis de la primera hipótesis indica que no se pueden aceptar, ya que no se encontró relación entre el nivel de comprensión de lectura y el uso de las estrategias de adquisición de la información en los alumnos del quinto gra- do de educación secundaria pertenecientes al distrito de Independencia. Resultados similares se observaron al analizar la relación en el nivel de comprensión de lectura y el uso de las estrategias de codificación, recuperación y apoyo al procesamiento de la información en estos estudiantes, donde las correlaciones entre ambas variables, en correspondencia a las respectivas hipótesis $(2,3,4)$, no fueron significativas, y además en algunos casos fueron negativas. Considerando que las estrategias de aprendizaje constituyen aquellos recursos cognitivos que el aprendiz utiliza con la intención de influir en los procesos de adquisición, codificación, recuperación y regulación de la infor- 
mación propia de la educación formal básica, debiera estar relacionada como lo menciona Román (2004), con aquellas actividades que permiten al alumno volverse un estudiante independiente, entre ellas la comprensión de lectura, ya que los alumnos de quinto grado de secundaria requieren un buen nivel de comprensión de lectura para poder aplicarlo en sus necesidades de desarrollo y lograr nuevos aprendizajes en los siguientes grados de instrucción, sean estos universitarios o técnicos.

Con relación al nivel de comprensión de lectura de los alumnos, se observó que el mayor porcentaje de estudiantes se ubica en un nivel deficitario, lo cual estaría indicando que estos estudiantes no cuentan con los prerrequisitos para la lectura y presentan serias dificultades para la comprensión del texto. Un menor porcentaje se ubica en el segundo nivel de comprensión, en el cual los sujetos, a pesar de lograr una comprensión global aproximada, se pierden en los detalles, lo que lleva a olvidar fácilmente lo comprendido y a requerir de apoyo pedagógico específico para el tipo de texto.

En el nivel independiente se ubican pocos casos de lectores autónomos, que comprendieron la lectura con fluidez y precisión, y entendieron la estructura íntegra del texto.

Dentro de la comprensión de lectura, se aprecia que el desempeño más bajo obtenido por los alumnos fue en el texto numérico, donde presentan difi- cultades para efectuar operaciones numéricas simples a partir de información contenida en textos impresos. Los resultados con base en el texto informativo permiten apreciar que estos estudiantes fracasan en la comprensión de libros y artículos periodísticos. En el caso del texto documentario, los estudiantes lograron mejores resultados en comparación con los textos anteriormente mencionados, pero cabe resaltar sin embargo que todos los textos se encuentran por debajo del nivel crítico (González \& Quesada, 1997). Los resultados en esta lectura evidencian que los alumnos no identifican ni aprovechan la información contenida en formularios, cuadros, gráficos e índices que acompañan el material para lograr la comprensión.

Estos resultados concuerdan con los hallazgos de la investigación realizada por González y Quesada en Lima (1997), quienes encontraron que los alumnos de secundaria tenían un rendimiento deficiente en los textos básicos e inclusive no alcanzaban el $25 \%$ de la puntuación posible en los textos complementarios, esto llevó a este autor a concluir que los estudiantes eran analfabetos funcionales, debido a que presentaban problemas lectores en el nivel de comprensión más que en la decodificación. También coinciden con los resultados obtenidos a través de la Evaluación Internacional para Alumnos Pisa (OCDE-Ince, 2001), que evidenció que el $80 \%$ de estudiantes calificó 
en el nivel 1, es decir, presentaron muy mala o nula internalización de lo leído.

En los niveles de las estrategias de aprendizaje, se observa que los alumnos se ubican mayoritariamente en el nivel intermedio de empleo de las estrategias de adquisición, codificación, recuperación y apoyo al procesamiento de la información.

Estos resultados coinciden con lo reportado por Escurra (2004) y Román y Gallego (1994), de manera que es factible afirmar que los escolares evaluados conocen y hacen uso de técnicas o tácticas en forma selectiva en algunas de las actividades de aprendizaje propias de cada área curricular.

Así, de acuerdo con los resultados obtenidos en el presente estudio se observa que los estudiantes del quinto grado de educación secundaria del distrito de Independencia se encuentran en niveles de comprensión muy bajos y la cantidad de lectores deficientes es muy alta, debido a que no poseen la capacidad de procesamiento que va de lo literal a lo inferencial. También se observa que, a pesar de conocer y usar estrategias para controlar sus propios procesos de aprendizaje en algunas actividades escolares, los adolescentes evaluados no saben o son ineficientes en la aplicación de las tácticas y recursos para guiar los procesos de adquisición, codificación, recuperación y monitoreo de la información para elaborar su comprensión y para controlar mejor sus procesos de aprendizaje a partir de la lectura, lo cual les impide ser lectores autónomos $\mathrm{y}$, por tanto, poder afrontar con esperanza de éxito las demandas de la modernización, el entorno cultural contemporáneo de carácter científico y tecnológico, y conducir los cambios necesarios para modificar sus condiciones de vida.

También se puede señalar que los alumnos demuestran haber desarrollado las estrategias de aprendizaje pero no saben seleccionar los propios recursos y técnicas para aplicarlas a la lectura; por lo tanto, los colegios de educación secundaria deberían diseñar programas para desarrollar la competencia lectora de los alumnos y la destreza de articular y seleccionar eficazmente las estrategias de adquisición, codificación, recuperación y de apoyo al procesamiento para lograr la comprensión de los textos. La implementación de estos programas requeriría de la competencia didáctica del docente, el currículo y las nuevas tecnologías de información.

\section{CONCLUSIONES}

Los resultados de la investigación permiten llegar a las siguientes conclusiones:

- No existe relación entre la comprensión de lectura y el uso de las estrategias de adquisición de la información en los alumnos del quinto grado de educación secundaria pertenecientes al distrito de Independencia. 
- No se establece relación entre la comprensión de lectura y el uso de las estrategias de codificación de la información en los alumnos.

- No existe relación entre la comprensión de lectura y el empleo de las estrategias de recuperación de la información en los alumnos.

- No existe relación entre la comprensión de lectura y el uso de las estrategias de apoyo al procesamiento de la información en los alumnos.

- En nivel deficitario de comprensión de lectura en función del tipo de lectura en el texto informativo se ubica el $67,3 \%$ de los alumnos, en el documentario el $58,0 \%$ y en el numérico el $78,9 \%$, lo que indica que estos estudiantes presentan serias dificultades para la comprensión del texto o no poseen los prerrequisitos para la lectura.

- En el segundo nivel (dependiente) en el texto del informativo se ubica el 30,5\% de estudiantes, en el texto documentario el $39,6 \%$ y en el numérico el $20,9 \%$. Estos alumnos requieren apoyo pedagógico específico para cada tipo de texto, pues comprenden de manera global y pierden detalles no comprendidos o los olvidan fácilmente.

- Solo el $2,1 \%$, el $2,4 \%$ y el $0,2 \%$ de los alumnos se ubican en el nivel independiente en los textos informati- vo, documentario y numérico, respectivamente. Es decir, realizan la lectura con fluidez y precisión.

- En función de las estrategias de adquisición de la información se ubican en el nivel superior el 19,6\%, en el nivel intermedio el $53,8 \%$ y en el nivel inferior el $26,6 \%$ de los estudiantes evaluados.

- En las estrategias de codificación de la información el 24,0\%, el 49,9\% y el $26,2 \%$ de estudiantes alcanzaron los niveles superior, intermedio e inferior, respectivamente.

- Con respecto a las estrategias de recuperación de la información pertenecen al nivel superior el $24.6 \%$, al nivel intermedio el 50,3\% y al nivel inferior el $25,1 \%$ de los evaluados.

- En estos escolares, el 25,3\% alcanzan el nivel superior, el 49,0\% el nivel intermedio y el $25,7 \%$ el nivel inferior en las estrategias de apoyo al procesamiento de la información.

\section{REFERENCIAS}

Barton, P. \& Kirsh, I. (1990). Workplace competencies: the need to improve literacy and employment readiness. Washington D.C.: Department of Education.

Beltrán, J. (1996). Estrategias de aprendizaje. En J. Beltrán \& C. Genovard (Eds.). Psicología de la instrucción. Variables y procesos básicos. Madrid: Síntesis. 
Cairney, T. (1992). La enseñanza de la comprensión lectora. Madrid: Ediciones Morata S.A.

Carroll, D. (2006). Psicología del lenguaje (4. ${ }^{\mathrm{a}}$ ed.). Madrid: Thomson.

Condemarín, M. \& Milicic, N. (1988). Test de Cloze. Aplicaciones Psicopedagógicas. Santiago de Chile: Editorial Andrés Bello.

Cuetos, F. (1991). Psicología de la lectura (2. ${ }^{a}$ ed.). Madrid: Escuela Española.

Cueto, S. (2007). Las evaluaciones nacionales e internacionales de rendimiento escolar en el Perú: Balance y perspectivas. En Investigación, políticas y desarrollo en el Perú. Lima: Grade, pp. 405-455.

Dirección Nacional de Educación Secundaria y Superior Tecnológica. (2006). Guía de Estrategias Metacognitivas para Desarrollar la Comprensión Lectora. Lima: Ministerio de Educación.

Escurra, M. (2002). Relación entre la comprensión de la lectura y la velocidad lectora en alumnos del sexto grado de primaria de centros educativos estatales y no estatales de $\mathrm{Li}$ ma metropolitana. Tesis para optar el grado de magíster (no publicada), Universidad Ricardo Palma, Lima, Perú.

Escurra, M. (2004). Influencia de las estrategias de aprendizaje y la refle- xión activa sobre el rendimiento escolar en los alumnos del quinto año de secundaria de la ciudad de Lima. Revista de Investigación en Psicología, 7(1), 51-80. Lima: Universidad Nacional Mayor de San Marcos.

García, J. (2001). Dificultades de aprendizaje e intervención psicopedagógica Lectura y Escritura. (2. ${ }^{a}$ ed.). Madrid: EOS.

Genovard, C. \& Gotzens, C. (1996). Psicología de la instrucción. Variables y procesos básicos. Madrid: Síntesis.

González, R. (1996). Lectoescritura: Aspectos cognitivos y evolutivos. Cuadernos Cedhum, 5. Lima: Sergrafin EIRL.

González, R. \& Quesada, R. (1997). Analfabetismo funcional en estudiantes de Lima. Revista de Psicología, 1(1), 79-90. Lima: Universidad Nacional Mayor de San Marcos.

Hernández, R.; Fernández C. \& Baptista, P. (2003). Metodología de la Investigación. (3. ${ }^{\mathrm{a}}$ ed.). México: McGraw-Hill.

Llece (2001). Primer estudio internacional comparativo sobre lenguaje, matemática y factores asociados, para alumnos del tercer y cuarto grado de la educación básica. Informe técnico. Santiago de Chile: Unesco. 
Monereo, C. (2000). Estrategias de aprendizaje. Madrid: Visor Dis. S.A.

Morles, A. (1999). El proceso de la comprensión en la lectura. Revista Latina de Pensamiento y Lenguaje, 279-293.

Nisbel, J. \& Schucksmith, J. (1987). Estrategias de Aprendizaje. Madrid: Santillana.

OCD-INCE. (2001). La medida de los conocimientos y destrezas de los alumnos. La evaluación de la lectura, las matemáticas y las ciencias en el Proyecto Pisa-2000/OCDE. Madrid: INCE.

Pinzás, J. (2003). Metacognición y lectura. Lima: Pontificia Universidad Católica del Perú.

Pramling, I. (1990). Learning to Learning: A Study of Swedish Presschool Children. Nueva York: SpringerVerlag.
Román, S. \& Gallego, R. (1994). ACRA-Escala de Estrategias de Aprendizaje (2. ${ }^{\mathrm{a}}$ ed.) Madrid: TEA Ediciones S.A.

Román, J. (2004). Procedimiento de aprendizaje autorregulado para universitarios: "La estrategia de lectura significativa de textos". Revista Electrónica de Investigación Psicoeducativa, 2(1), 113-132.

Sánchez, H. \& Reyes, C. (2002). Metodología y diseños en la investigación científica. Lima: Editorial Universitaria.

Solé, I. (1999). Estrategias de lectura. Barcelona: GRAÓ.

Tapia, V. (2008). Procesos cognitivos y desempeño lector. Revista de Investigación en Psicología, 11(1), 3768. Lima: Universidad Nacional Mayor de San Marcos. 